\title{
Robust control of uncertain structures
}

\author{
Paolo Venini \\ Department of Structural Mechanics, University of Pavia, Via Ferrata 1, I-27100 Pavia, Italy
}

\begin{abstract}
Objective of this paper is to present robust control strategies able to handle acceleration feedback. The robustness of the controller is investigated in respect to both stability and performance measures. The uncertainty set is chosen as a convex one with either real or complex structured uncertainty. $H_{\infty}$ control is firstly used to comply with the stability requirements whereas a $\mu$ synthesis approach is then adopted as far as the performance is concerned. The degradation of the performance in respect to the size of the perturbation is eventually proposed as a merit parameter to rank different controllers. Numerical simulations are proposed on a MDOF structural system under earthquake excitation. (C) 1998 Elsevier Science Ltd. All rights reserved.
\end{abstract}

\section{Introduction}

Analyzing uncertain structural systems has been a major topic of research for a few decades within the civil engineering community. Uncertainties in the acting loads as well as in the physical parameters that define a structure may actually lead to an extremely poor operating behavior, especially if the sources of uncertainty are not considered during all the phases of the design. This is even more important when the system is equipped with active devices that may induce instability in the plant dynamics. A rationale scheme for designing robust controllers therefore requires:

1. the selection of a suitable approach for simulating uncertainties,

2. a measure of optimality to be complied with by the controller,

3. a theoretical/numerical scheme for the actual computation,

4. some a-posterior verifications of the behavior of the system under control.

All these listed items are investigated in this paper. Uncertainty is introduced via convex idealizations, i.e. a norm-bounded convex set to which the system to be controlled belongs is introduced. No system realization is more probable than the other, actually probability is not introduced at all. Both stability and performance goals are sought by designing a controller via $H_{\infty}$ theory. The robustness of the design is attained thanks to the adoption of a $\mu$ synthesis approach that is numerically implemented via the iterative scheme known as D-K. Numerical simulations on a three degree-of-freedom system under earthquake noise are worked out confirming the achievement of robust stability and performance. Feedback of accelerations is considered as a more realistic alternative to classic state-space feedback methods that are seldom realizable in practice.

\section{Uncertainty modeling}

\subsection{Preliminaries}

Different models of uncertainties may be adopted to perform a control synthesis able to account for deviation of the system from its nominal values. The one adopted herein is based on convex models that is the nominal plant is generated via prescribed values of system parameters that may take any value within a prescribed "ball" centered at the nominal values and of given radius. With robustness of a property we will mean that for all possible values of the parameters within this convex region the property is met by the controller. Central in the uncertainty model adopted is 


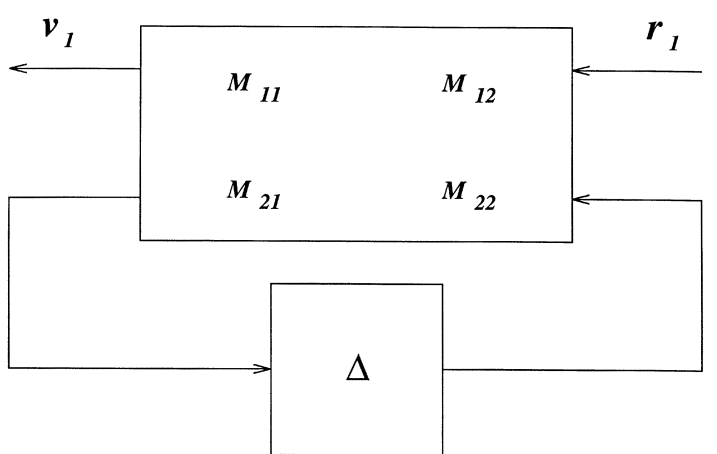

Fig. 1. Lower linear fractional transformation.

the concept of linear fractional transformation (LFT) that is recalled next.

\subsection{Linear fractional transformations}

Assume the relationships

$v_{1}=M_{11} r_{1}+M_{12} r_{2} \quad v_{2}=M_{21} r_{1}+M_{22} r_{2}$

hold for the vectors $v=\left\{\begin{array}{ll}v_{1} & v_{2}\end{array}\right\}^{\prime}$ and $r=\left\{\begin{array}{ll}r_{1} & r_{2}\end{array}\right\}^{\prime}$. Furthermore, a matrix $\Delta$ is supposed to relate $v_{2}$ to $r_{2}$, i.e. $r_{2}=\Delta v_{2}$. The LFT of $M$ by $\Delta$ is then computed by closing the lower loop as in Fig. 1.

An explicit relationship between $v_{1}$ and $r_{1}$ may then be derived by condensing out $v_{2}$ and $r_{2}$ obtaining

$v_{1}=\left[M_{11}+M_{12} \Delta\left(I-M_{22} \Delta\right)^{-1} M_{21}\right] r_{1}=F_{\mathrm{L}}(M, \Delta) r_{1}$,

where a subscript $\mathrm{L}$ indicates the lower loop closure of $M$ by $\Delta$. In a similar way one may of course define the upper loop closure of a plant matrix with a perturbation matrix. It is now shown how LFT plays a crucial role for uncertainty modeling in either structured and unstructured idealizations.

\subsection{Structured parametric uncertainty}

Suppose a given structural parameter, e.g. a mass or stiffness, is known to vary between prescribed bounds. If $p$ denotes such parameter and $p_{1}$ and $p_{\mathrm{u}}$ its lower and upper bounds, i.e. if the inequalities

$p_{1} \leq p \leq p_{\mathrm{u}}$

hold true, it is useful to derive an expression of $p$ in terms of its mean value $\bar{p}$ (not in probabilistic sense but intended simply as the average of its lower and upper bounds) and of the dispersion of it around $\bar{p}$, i.e. $\sigma_{\mathrm{p}}=\bar{p}-p_{1}$. It should be again pointed out that no probability is used here but rather a convex model of uncertainty within a metric design space. One may easily verify that $p$ may be written as
$p=F_{\mathrm{L}}\left(\left[\begin{array}{cc}\bar{p} & \sigma_{\mathrm{p}} \\ 1 & 0\end{array}\right], \delta\right)=F_{\mathrm{L}}\left(M_{\mathrm{p}}, \delta\right)$,

where $\delta \in[-1,1]$. Any time the parameter $p$ appears within a loop, one simply has to replace it with $F_{\mathrm{L}}$ $\left(M_{\mathrm{p}}, \delta\right)$. In the same way one may show that the inverse of $p$ is governed by a similar expression. Thanks to this model, an uncertain plant may be cast within classical state-space frameworks simply because the uncertainty model adopted satisfies a standard input/output relationship the transfer function of which is recovered thanks to LFT. Therefore, uncertainties in masses, damping coefficients, natural frequencies and so on may be studied via the above model.

\subsection{Neglected dynamics}

Another source of uncertainty, not of parametric type, is represented by unmodelled dynamics, especially in the high frequency range. A linear continuous system is actually characterized by an infinity of eigenmodes and associated eigenfrequencies. When a discretization process is used, e.g. a finite-element scheme, higher modes are usually neglected so that one studies a truncated version of the actual model. To include such neglected dynamics the following model may be adopted. It calls for the definition of

- a nominal plant, say $G(s)$;

- a multiplicative uncertainty weighting function, say $W_{\mathrm{u}}(s)$ (see Fig. 2).

Then, one may define a set of plants, say $\mathscr{M}\left(G, W_{\mathrm{u}}\right)$, as

$\mathscr{M}\left(\mathscr{G}, \mathscr{W}_{\mathrm{u}}\right)=\left\{\mathscr{G}:\left|\frac{\mathscr{G}(j \omega)-\mathscr{G}(j \omega)}{\mathscr{G}(j \omega)}\right| \leq\left|\mathscr{W}_{\mathrm{u}}(j \omega)\right|\right\}$

with the technical restriction that the number of righthalf plane poles of $\tilde{G}$ be equal to that of $G$. Therefore, at each frequency $\omega, W_{\mathrm{u}}(j \omega)$ represents the maximum difference in percent between the nominal plant $G$ and the perturbed one $\tilde{G} . \mathscr{M}\left(G, W_{\mathrm{u}}\right)$ is easily seen to be a convex set (actually a ball) centered at $G$. The nice feature of this approach is that $W_{\mathrm{u}}$ is selected by the analyst. If the objective is to include higher order dynamics not explicitly considered in the equations of motion then $W_{\mathrm{u}}$ will be an increasing function of $\omega$ so that the deviation from the nominal plant is potentially higher at larger frequencies. The rest of this section is devoted to showing how the proposed model may be framed within the LFT scheme previously presented. Let

$\Delta=\frac{\tilde{G}-G}{G W_{\mathrm{u}}}$, 


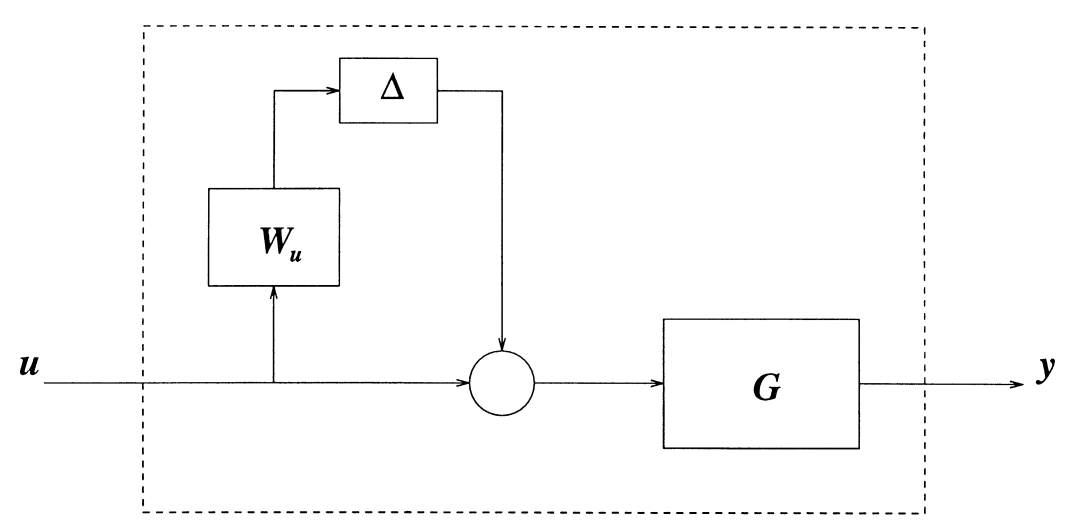

Fig. 2. Plant with multiplicative uncertainty.

with the additional constraint $\max _{\omega}|\Delta(j \omega)| \leq 1$. Then, if $y=G u$ is the nominal plant, $y=F_{\mathrm{U}}\left(H_{\text {mult }}, u\right)$ is any plant belonging to $\mathscr{M}\left(G, W_{\mathrm{u}}\right)$, where

$\left\{\begin{array}{l}z \\ y\end{array}\right\}=\left[\begin{array}{cc}0 & W_{\mathrm{u}} \\ G & G\end{array}\right]\left\{\begin{array}{l}w \\ u\end{array}\right\}=H_{\text {mult }}\left\{\begin{array}{l}w \\ u\end{array}\right\}$

(see Fig. 3).

\section{The structured singular value}

After we have presented a few models of uncertainty, the structured singular value theory is introduced, the final goal being the derivation of robust stability/performance criteria. In the most general framework the uncertainty set $\Delta$ is introduced as

$\Delta=\left\{\operatorname{diag}\left[\delta_{1} I_{r_{1}}, \ldots, \delta_{S} I_{r_{S}}, \Delta_{1}, \ldots, \Delta_{F}\right]: \delta_{i} \in C\right.$,

$$
\left.\Delta_{j} \in C^{m_{j} \times m_{j}}\right\} \subset C^{n \times n},
$$

where $S$ is the number of scalar blocks and $F$ that of complex full blocks. The uncertainty set we are going to use is a norm-bounded subset of $\Delta$, say $B_{\Delta}$, and is defined as
$B_{\Delta}=\{\Delta \in \Delta: \bar{\sigma} \leq 1\}$,

where $\bar{\sigma}$ denotes the maximum singular value. For a given matrix $M \in C^{n \times n}$, we can now define the structured singular value $\mu$ as

$\mu_{\Delta}(M)=\frac{1}{\min \{\bar{\sigma}(\Delta): \Delta \in \Delta, \operatorname{det}(I-M \Delta)=0\}}$

with the only exception $\mu_{\Delta}=0$ when $I-M \Delta$ turns out to be nonsingular for all $\Delta \in \Delta$. A physical interpretation of $\mu_{\Delta}$ may be obtained by taking a look to Fig. 4 . The algebraic relationships shown may be written as

$u=M v, \quad v=\Delta u$

It is easy to see that $\operatorname{det}(I-M \Delta)=0$ is the necessary condition for the existence of nontrivial solutions to Eq. (11). Therefore, $\mu_{\Delta}(M)$ is a measure of the smallest $\Delta$ that causes instability of the system. As to the computation of $\mu_{\Delta}$, the following bounds may be established

$\rho(M) \leq \mu_{\Delta} \leq \bar{\sigma}(M)$

in which $\rho$ denotes the spectral radius. Unfortunately,

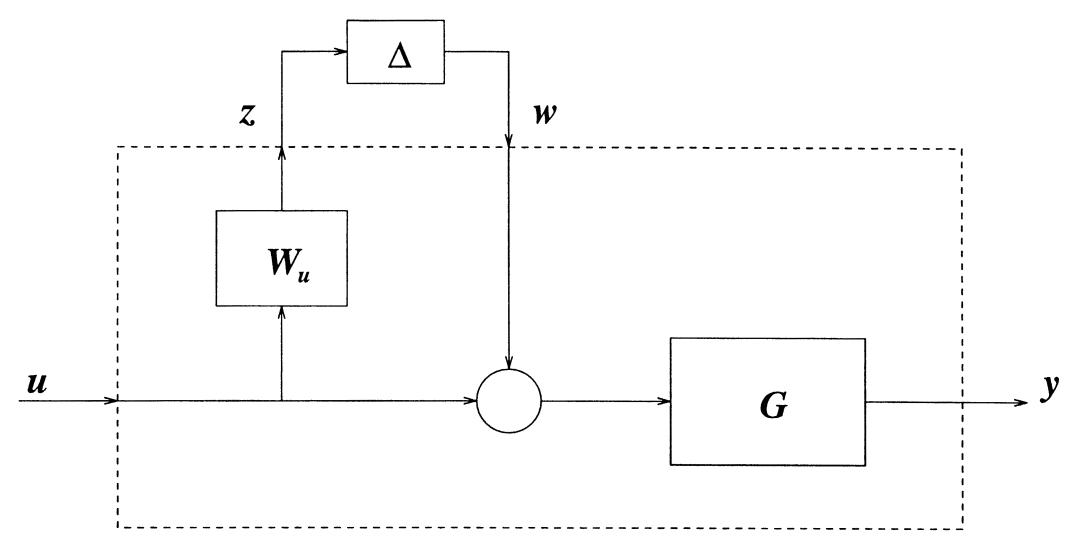

Fig. 3. Multiplicative uncertainty as a LFT. 


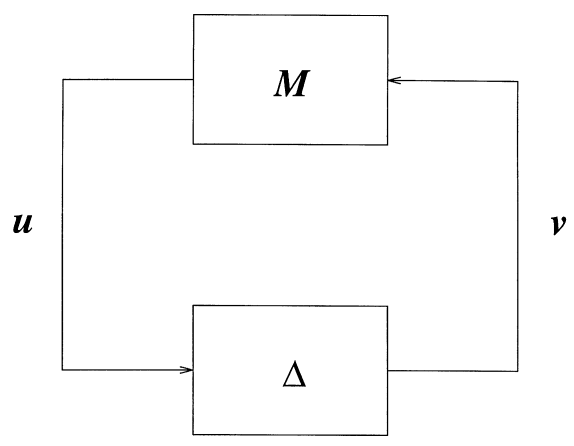

Fig. 4. Feedback interpretation of $\mu$.

these bounds do not allow an accurate approximation of $\mu_{\Delta}$ since $\rho$ and $\bar{\sigma}$ may be far from each other. The trick is then to introduce some linear transformation that causes $\rho$ and $\bar{\sigma}$ to come closer but leaves $\mu$ unchanged. To this goal two subsets of $C^{n \times n}$ are introduced as

$Q_{\Delta}=\left\{Q \in \Delta: Q^{*} Q=I_{n}\right\}$

and

$D_{\Delta}=\left\{\begin{array}{c}\operatorname{diag} \quad\left[D_{1}, \ldots, D_{\mathrm{S}}, d_{1} I_{m_{1}}, \ldots, d_{F-1} I_{m_{F-1}}, I_{m_{F}}\right] \\ D_{i} \in C^{r_{i} \times r_{i}}, D_{i}=D_{i} *>0, d_{j} \in R^{+}\end{array}\right\}$,

where a starred matrix denotes its conjugate transpose. One may then show that [5] the following tightened bounds hold

$\max _{Q \in Q_{\Delta}} \rho(Q M) \leq \mu_{\Delta} \leq \inf _{D \in D_{\Delta}} \bar{\sigma}\left(D M D^{-1}\right)$.

The lower bound may be shown to be an equality but is not computationally viable since the function $\rho(Q M)$ is characterized by a large amount of local minima that cannot be escaped from by any numerical optimization technique. On the other side, finding the upper bound amounts to solving a convex problem that will yield in principle a unique solution. Unfortunately, however, such extremum point need not be the same as $\mu$, i.e. the upper bound inequality is in fact such. The relationship between the upper bound and $\mu$ depends upon the selected uncertainty model. In particular, when $2 S+F>3$ one has always equivalence between $\mu$ and inf. The technique known as $\mathrm{D}-\mathrm{K}$ iteration is based on successive refinements of the upper bound in Eq. (15). The D-K iteration is reviewed in the following subsection.

\subsection{Fundamentals of $D-K$ iteration}

The objective of $\mu$-synthesis is to minimize the peak value of $\mu_{\Delta}$ over all stabilizing controllers. In formulae, one has to solve the optimization problem

$\min _{K} \max _{\omega} \mu_{\Delta}\left(F_{\mathrm{L}}(P, K)(j \omega)\right)$.

Thanks to Eq. (15), i.e. by substituting $\mu$ with its upper bound, a new convex optimization problem is set forth as

$$
\begin{aligned}
& \min _{K} \min _{D, D_{\omega} \in D_{\Delta}} \max _{\omega} \bar{\sigma}\left[D_{\omega} F_{\mathrm{L}}(P, K)(j \omega) D_{\omega}^{-1}\right] \\
& =\min _{K} \min _{D, D_{\omega} \in D_{\Delta}}\left\|D F_{\mathrm{L}}(P, K) D^{-1}\right\|_{\infty},
\end{aligned}
$$

where the notation $D, D_{\omega} \in D_{\Delta}$ means that for each fixed $\omega, D_{\omega}$ belongs to $D_{\Delta}$. The solution to the optimization problem in Eq. (17) is searched via a two step iterative approach referred to as $\mathrm{D}-\mathrm{K}$ iteration. The initialization is usually made by choosing $D$ as the identity matrix. The $\mathrm{D}-\mathrm{K}$ iteration goes as follows.

\subsubsection{STEP I: D fixed}

Eq. (17) is solved for given $D$ matrices. In this case, one has therefore to solve a classical $H_{\infty}$ problem that has as system matrix $P_{\mathrm{D}}$ rather than $P$ (see Fig. 5). The solution to this problem may therefore be found via classical methods in control literature and applicative software [1].

\subsubsection{STEP II: K fixed}

Once a candidate controller has been computed, it is kept fixed and the optimization is done with respect to $D$. The solution, i.e. the optimal $D$, is then approximated by $\hat{D}$ that is the matrix that is closest to $D$ within the class of stable, minimum phase, real-rational transfer functions. For more details, theoretical as well as numerical, we refer to [1].

\section{Robustness and $\mu$}

We are now ready to state a few conditions for stability and performance robustness, relate them to $\mu$ and
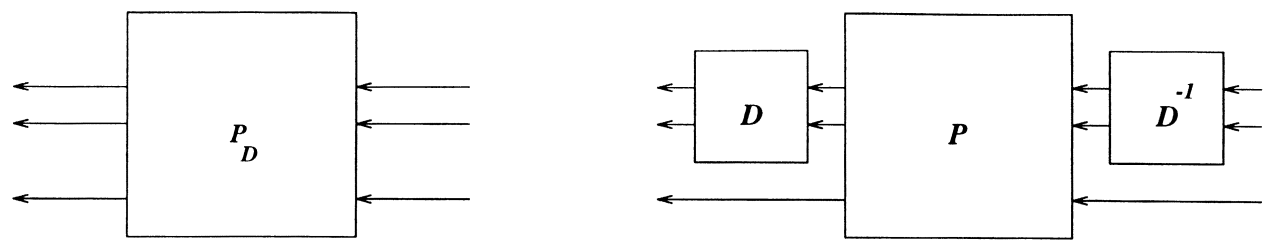

Fig. 5. $H_{\infty}$ optimization problem with D-K iteration. 
calculate a controller complying with the requisites that ensure robustness thanks to an iterative procedure named in fact $\mu$-synthesis in the literature.

\subsection{Notions of stability and performance robustness}

Nominal stability is a concept that is well known and dates back to Lyapunov. It may be checked by viewing at the real part of the eigenvalues of the closed-loop plant that has to be negative. The notions of nominal performance, robust stability and robust performance are given next. Given is a set of plants, say $\mathscr{G}$, defined as

$\mathscr{G}=\left\{\mathscr{G}_{\text {nom }}\left(\mathscr{I}+\Delta_{\mathrm{G}} \mathscr{W}\right): \Delta_{\mathrm{G}}\right.$ stable, $\left.\left\|\Delta_{\mathrm{G}}\right\| \leq\right\}$,

in which the weight $W$ shapes the uncertainty that is usually frequency-dependent. One has

- nominal performance is achieved if the condition

$\left\|W_{\mathrm{P}}\left(I+G_{\text {nom }} K\right)^{-1}\right\|_{\infty}<1$

holds true. In Eq. (19), $W_{\mathrm{P}}$ is again a weight.

- robust stability is achieved if, for all $G \in \mathscr{G}$ one has

$\left\|W K G_{\mathrm{nom}}\left(I+G_{\mathrm{nom}} K\right)^{-1}\right\|_{\infty}<1$

robust performance is achieved if, for all $G \in \mathscr{G}$, the closed-loop system is robustly stable and

$\left\|W_{\mathrm{P}}(I+G K)^{-1}\right\|_{\infty}<1$.

Nominal performance is achievable by means of standard $H_{\infty}$ design methodologies that are not dealt with herein and may be found in [3] among others. Attention is firstly focused on the robust stability problem that is tackled by considering the LFT in Fig. 6 . It is there shown the closed loop-plant $M=F_{\mathrm{L}}(H, K)$ that is acted upon by the uncertainty block $\Delta$. For this configuration the structured singular value $\mu_{\Delta}(M(j \omega))$ is computed as well as the supremum across $\omega$ that is denoted by $\beta$. Then robust performance is achieved for all perturbations that satisfy $\max _{\omega} \bar{\sigma}[\Delta(j \omega)]<1 / \beta$. In other words, the indicator
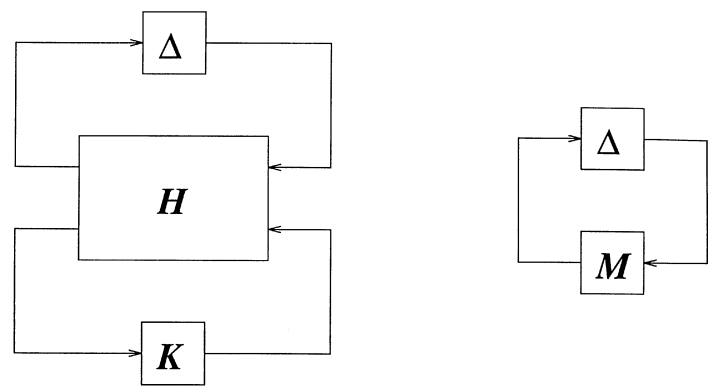

Fig. 6. The plant with feedback controller and uncertainty loop.

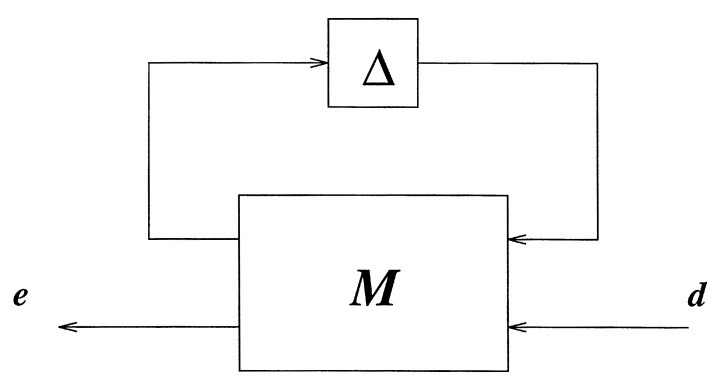

Fig. 7. Scheme for computing robust performance.

$\frac{1}{\max _{\omega} \mu_{\Delta}(M(j \omega))}$

is a stability margin with respect to the set $\Delta$ that acts upon the closed-loop plant $M$. It is to be noticed that disturbances do not enter the stability analysis that is investigated operating on the autonomous system. Conversely, the robust performance analysis calls for the re-introduction of disturbances and controlled outputs (errors) as in Fig. 7. Obviously, a performant controller is one that causes the closed-loop transfer function $T$ from $d$ to $e$ to be small, i.e. one imposes a condition of type

$\|T\|_{\infty} \leq 1$,

where of course $T=F_{\mathrm{U}}(M, \Delta)$. Thus, robust performance is achieved if and only if robust stability is achieved and also the condition in Eq. (23) met for all perturbations $\Delta \in \Delta$. The objective is now to solve the robust performance problem. The key idea is the equivalence between a problem of performance and an adjoined one of stability as shown in Fig. 8 [1]. Since in our case $T=F_{\mathrm{U}}(M, \Delta)$, robust performance is achieved if and only if the robust stability test for the augmented perturbation matrix

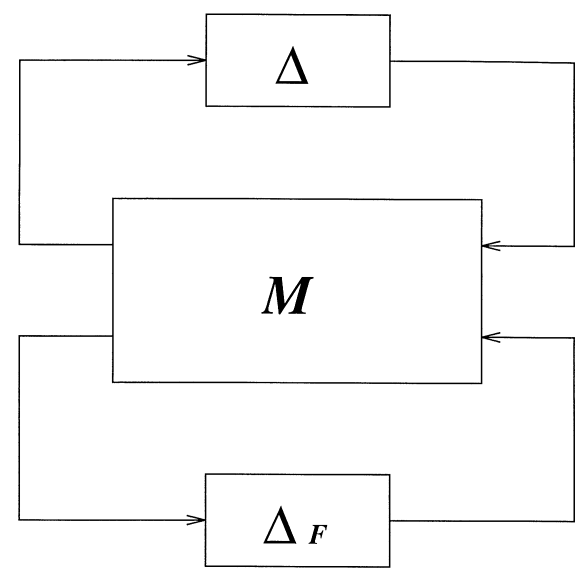

Fig. 8. Equivalence between performance and robustness. 


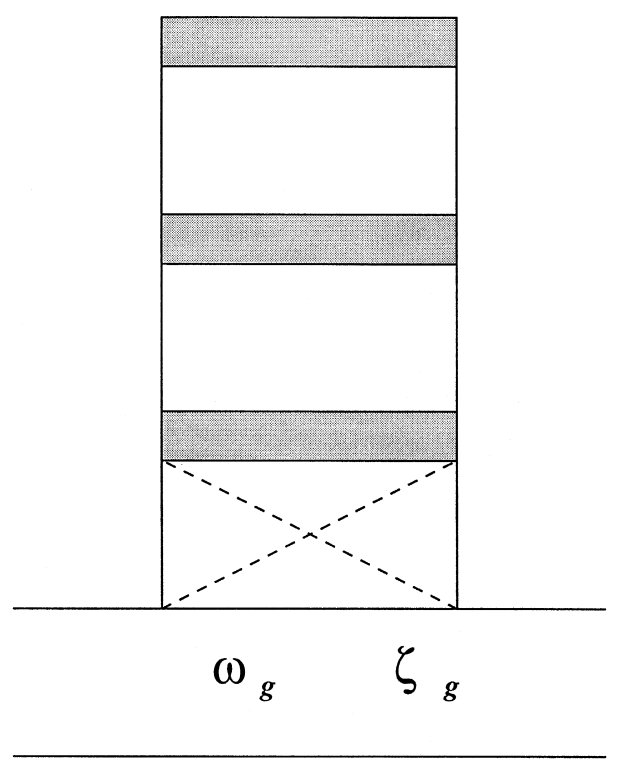

Fig. 9. Schematic of the structure under control.

$\Delta=\left[\begin{array}{cc}\Delta & 0 \\ 0 & \Delta_{F}\end{array}\right]$

is satisfied.

\subsection{Worst case performance}

A further merit parameter that allows one to judge about the performance of different controllers is given by the worst case performance degradation (WCPD). In simple words, the WCPD says whether the degradation of the performance of the controller due to perturbations of increasing size is smooth or not. The former case is of course the one should be attained whereas the latter should prevent the use of that controller in operating conditions. Mathematically, for a given perturbation size $\alpha$, WCPD is defined as

$$
\mathscr{W}(\mathscr{M}, \Delta, \alpha)=\max \underset{\max _{\omega} \bar{\sigma}[\Delta(j \omega)] \leq \alpha}{\Delta\left[\mathscr { F } _ { \mathscr { U } } \left(\mathscr{M}, \Delta \|_{\infty} .\right.\right.}
$$

In the numerical study section different controllers will actually be graded thanks to this merit parameter.

\section{Acceleration feedback structural control}

Objective of this section is to write the canonical triplet of equations governing the system dynamics in the presence of acceleration feedback. The state equation, the output (error) equation and the feedback (measurable) equation are to be written. Let the equation of motion in Lagrangean coordinates be

$M \ddot{\eta}+C \dot{\eta}+K \eta=E_{1} w+E_{2} u$

where $M, C$ and $K$ are the mass, damping and stiffness matrix, respectively, and $\eta$ is a generalized displacement vector. From Eq. (26), one may then get the acceleration vector $\ddot{\eta}$ as

$$
\begin{aligned}
\ddot{\eta}= & -M^{-1}[K \eta+C \dot{\eta}]+M^{-1} E_{1} w+M^{-1} E_{2} u \\
& =\left[-M^{-1} K-M^{-1} C\right] x+M^{-1} E_{1} w+M^{-1} E_{2} u(27)
\end{aligned}
$$

that fits into the usual framework [2]

$$
\left\{\begin{array}{c}
\dot{x}=A_{P} x+B_{w} w+B_{u} u, \\
z=C_{x} x+D_{z w} w+D_{z u} u, \\
y=C_{y} x+D_{y w} w+D_{y u} u,
\end{array}\right.
$$

with the correspondences

$$
\left\{\begin{array}{c}
C_{y}=\left[-M^{-1} K-M^{-1} C\right], \\
D_{y w}=M^{-1} E_{1}, \\
D_{y u}=M^{-1} E_{2} .
\end{array}\right.
$$

\section{Numerical study}

A three-degree-of-freedom lumped-parameter system is considered that is sketched in Fig. 9. Table 1 presents the undamped natural frequencies of the system along with the damping ratios. It is seen that the inherent damping of the system is significant but not sufficient and therefore an active device is added to damp out vibrations induced by external disturbances. The disturbance is the well known El Centro record that is passed through a Kanai-Tajimi filter $\left(\omega_{\mathrm{g}}=10 \mathrm{rad} / \mathrm{s}\right.$ and $\left.\xi_{\mathrm{g}}=0.5\right)$ before acting on the structure. The actual plant was shaped according to the multiplicative uncertainty scheme previously described and the output (error) was shaped as well. The relevant weighting functions are shown in Fig. 10. Notice that stability and performance weights are rather competitive, i.e. they cross each other rather close to the critical value of one [4]. The first and last controllers resulting from the $\mathrm{D}-\mathrm{K}$ iteration are given in Fig. 11 that shows how at first only robust stability was achieved whereas the final controller meets the robust performance requirement as well. The transfer function from the

Table 1

Physical properties of the system

\begin{tabular}{lll}
\hline MODE [-] & Frequency $[\mathrm{rad} / \mathrm{s}]$ & damping $[-]$ \\
\hline 1 & 2.39 & 0.0205 \\
2 & 5.90 & 0.0503 \\
3 & 8.70 & 0.0726 \\
\hline
\end{tabular}


P. Venini / Computers and Structures 67 (1998) 165-174

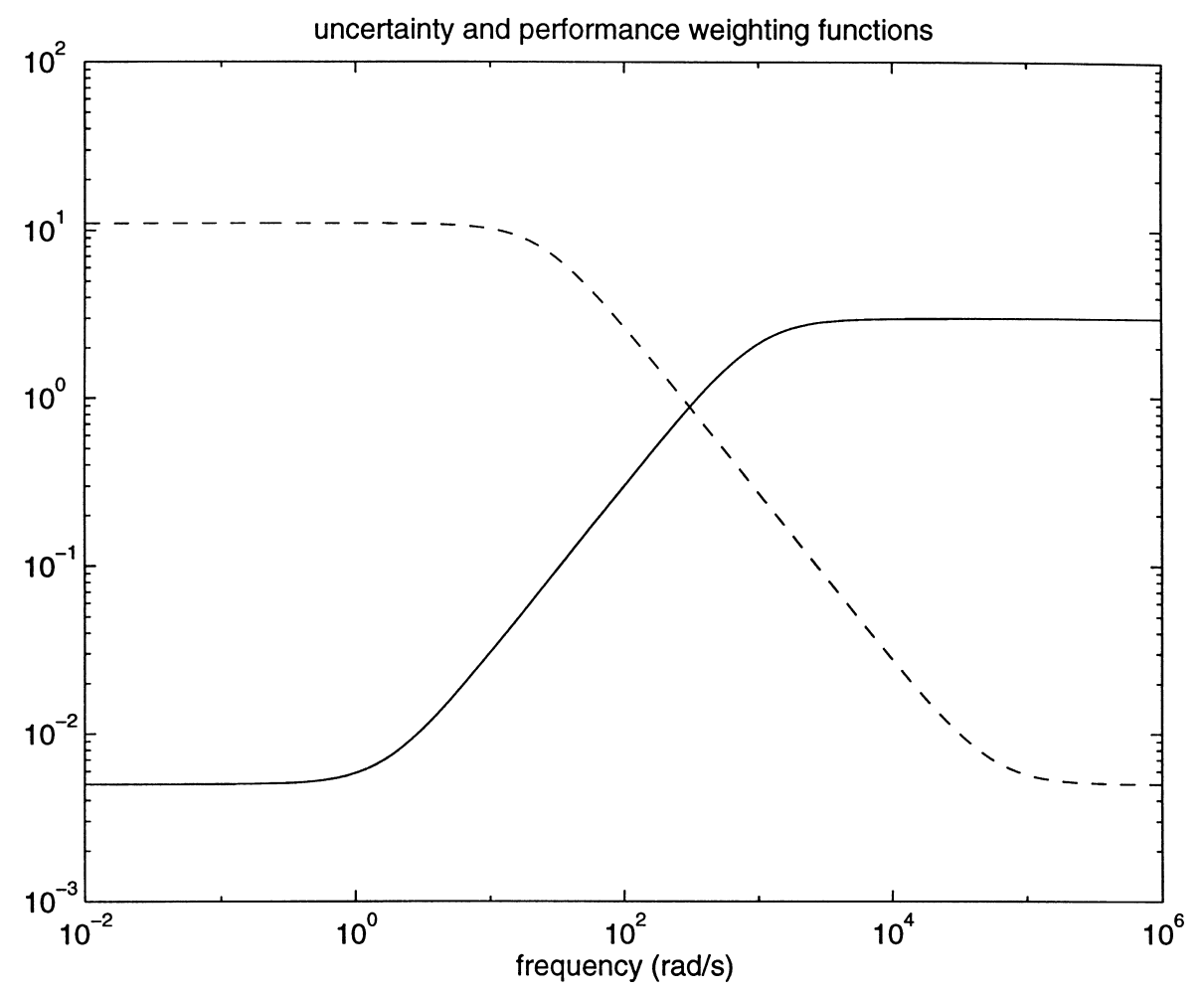

Fig. 10. Weighting functions for robust stability and performance.
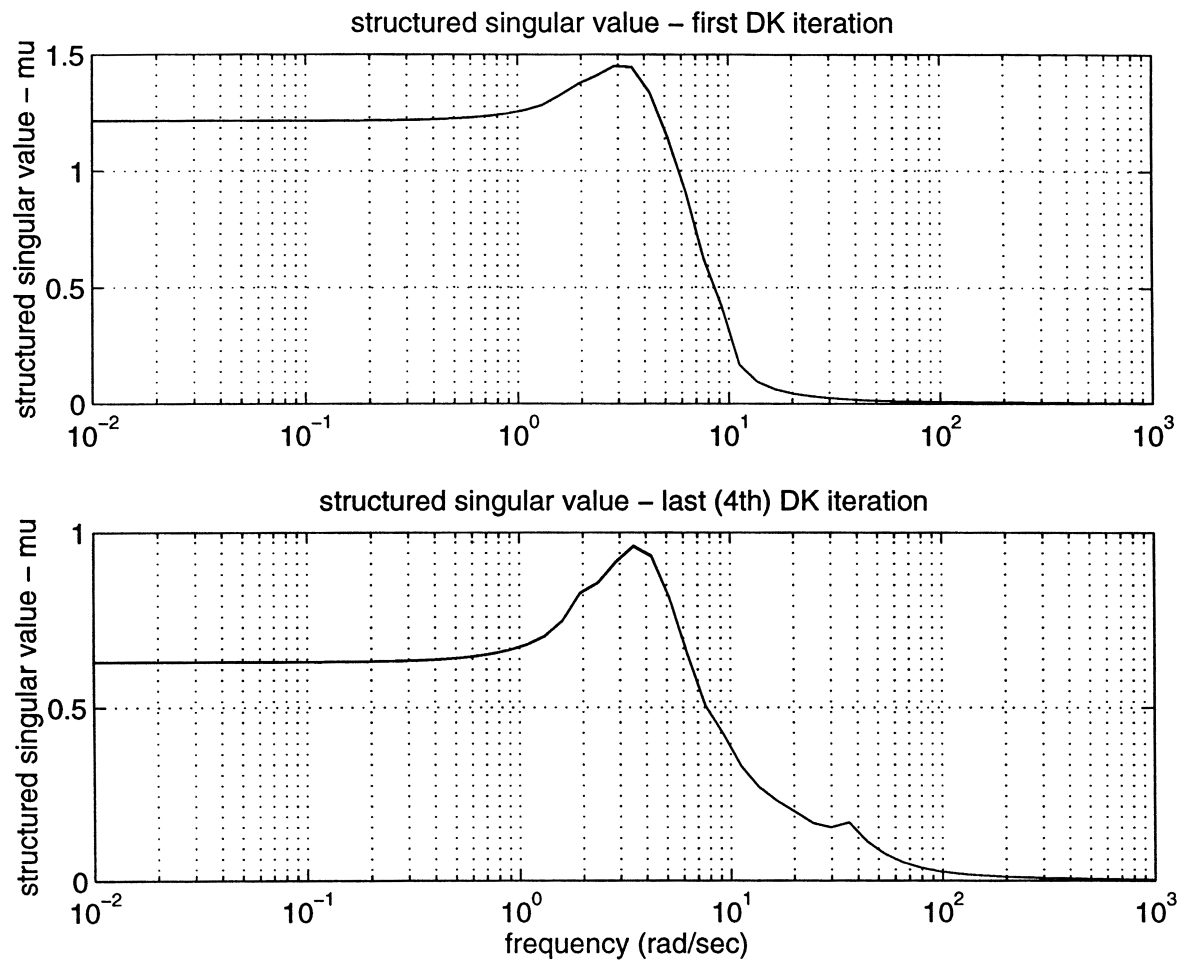

Fig. 11. The structured singular value vs D-K iteration. 

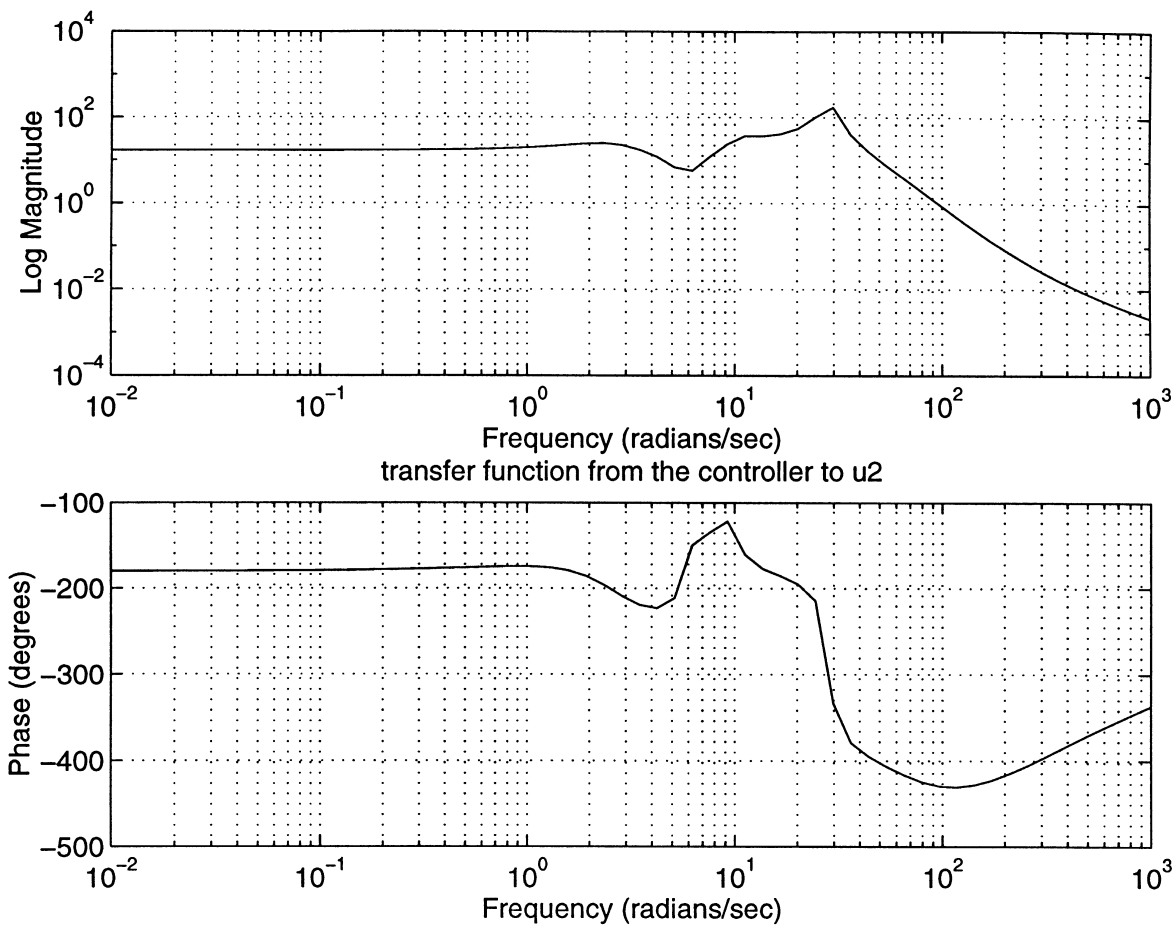

Fig. 12. Controller transfer function.
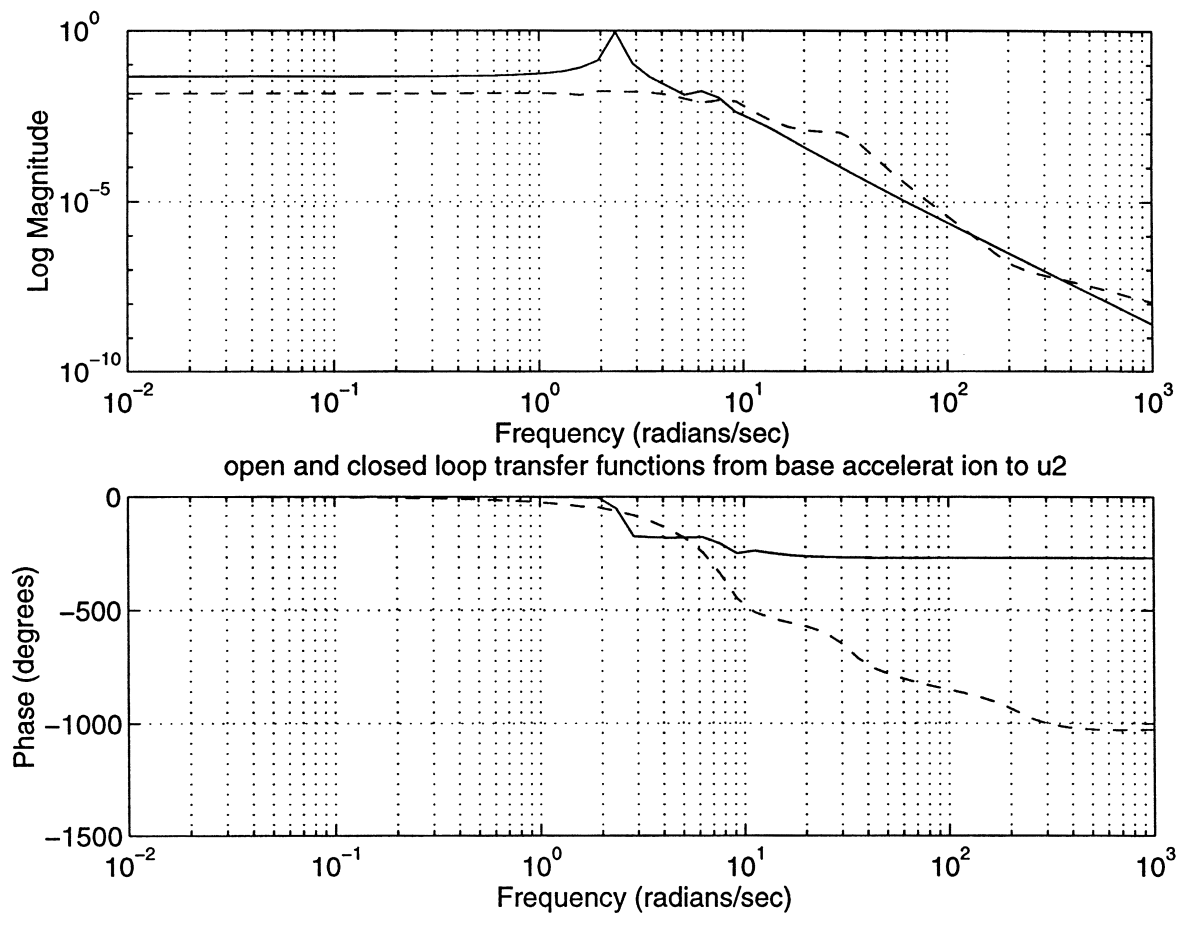

Fig. 13. Open and closed loop transfer function. 

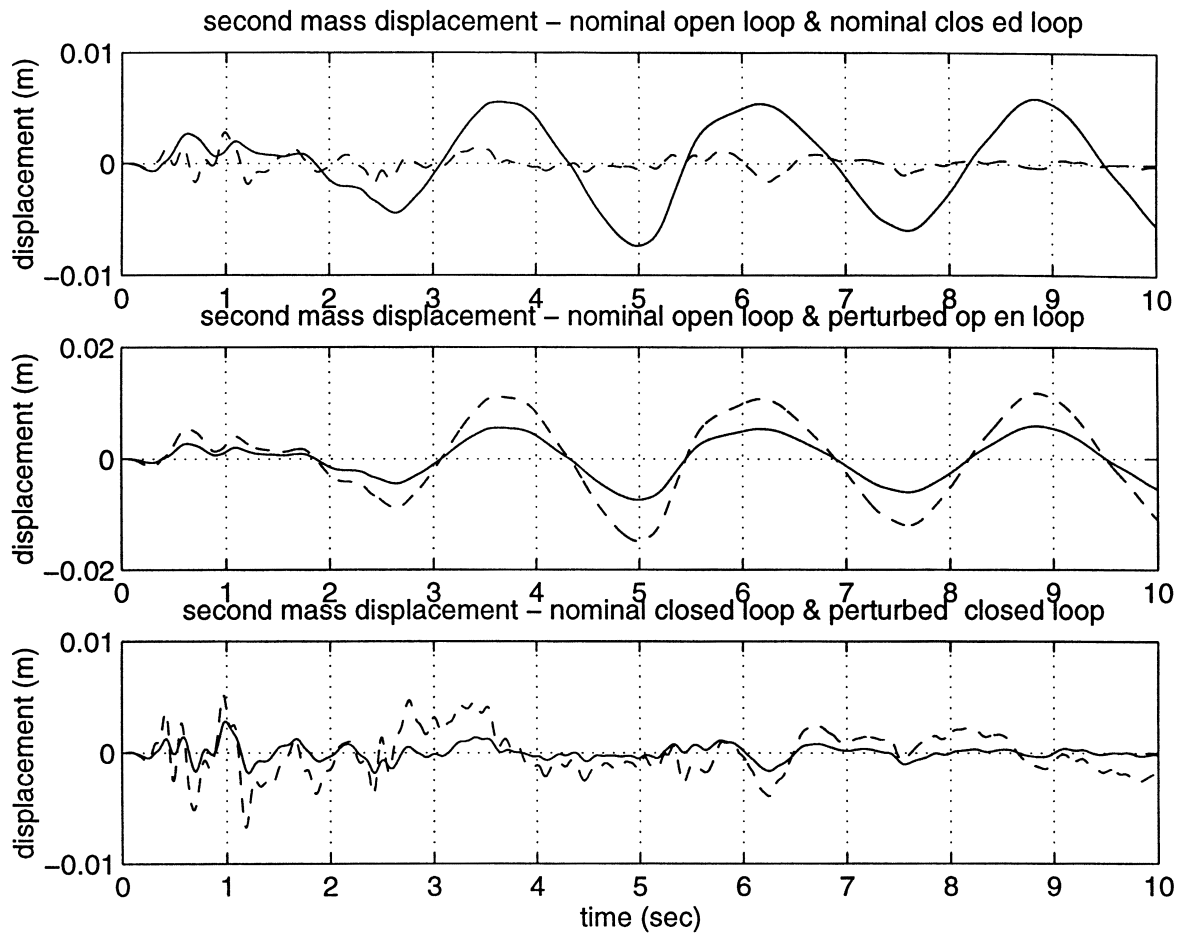

Fig. 14. Second floor displacement time histories.

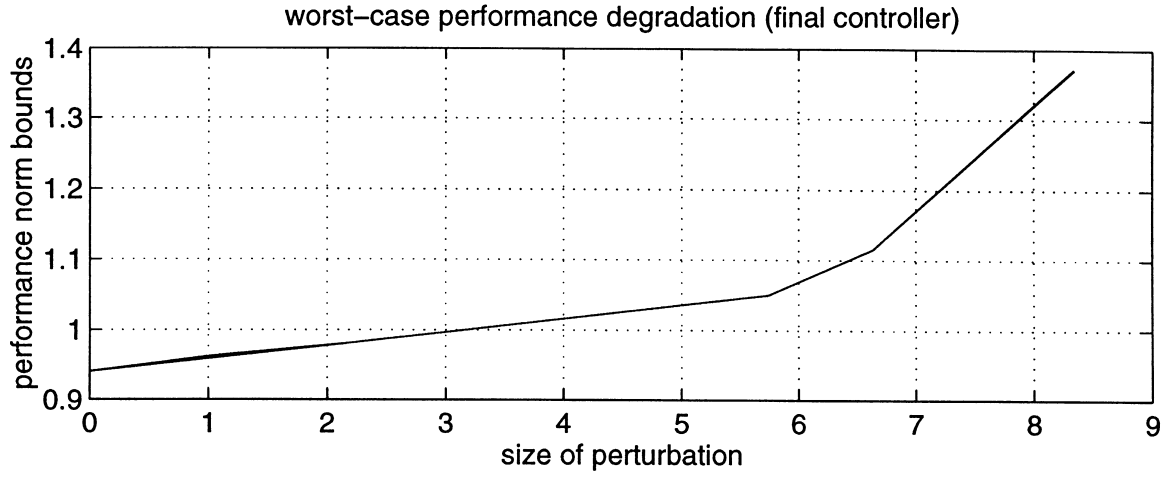

worst-case performance degradation (canonical $\mathrm{H} \_$infinity co ntroller)

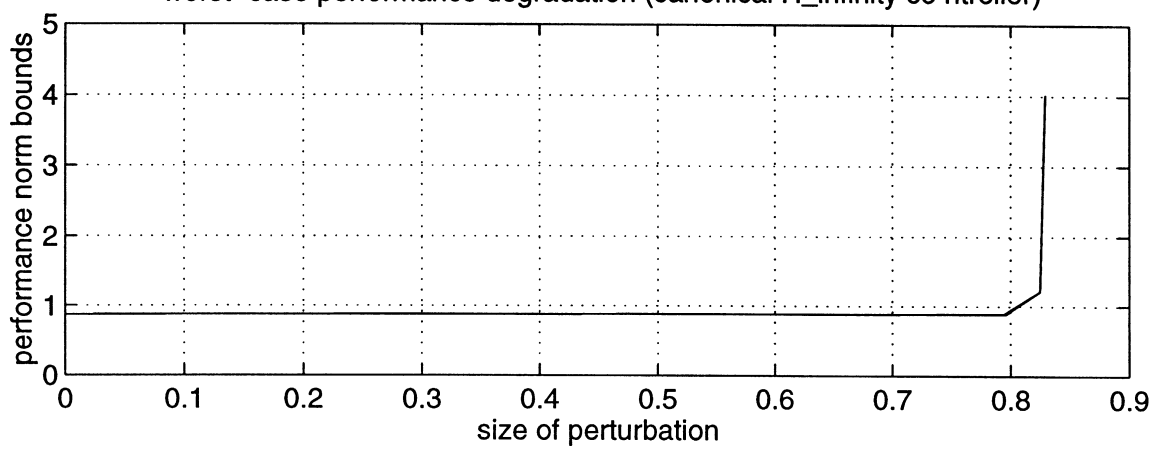

Fig. 15. Performance degradation vs size of perturbation. 
final controller to the displacement of the second mass is given in Fig. 12. Yet the second mass is investigated in Fig. 13 where a comparison between the open and closed loop transfer function from the disturbance to the displacement is presented. Fig. 14 is quite interesting in that it simulates the dynamics of the open and closed loop system in the nominal situation as well as in the case a perturbation of 0.5 is present in both the disturbance and control channels. Robust performance is clearly achieved. Finally, Fig. 15 presents the worstcase performance degradation for the $\mathrm{D}-\mathrm{K}$ controller as well as for a canonical $H_{\infty}$ one, designed based on the nominal plant without any shaping filter. The former is a smooth curve indicating that degradation takes place nicely, whereas the latter presents a sharp peak for a perturbation size that is one tenth of the former.

\section{Conclusions}

A $\mu$ synthesis approach has been presented for the active control of structural systems in the presence of uncertainties. Parametric uncertainties as well as nonparametric ones were considered and a controller designed capable of handling both of them and ensur- ing robust stability and robust performance. Acceleration feedback was considered instead of more classic state-feedback techniques. Ongoing extensions include designing a controller for elasto-plastic systems. The nominal design is in that case done with respect to the initial elastic properties and stiffness drops due to plasticity are modelled as structured uncertainties.

\section{References}

[1] Balas, G. J., Doyle, J. C., Glover, K., Packard, A. and Smith, R. $\mu$-analysis and synthesis toolbox (for use with MATLAB). The Math Works, Natick, MA.

[2] Boyd, S. P. and Barratt, C. H. (1991). Linear Controller Design: Limits of Performance. Prentice Hall Information and System Sciences Series.

[3] Doyle J C, Glover K, Khargonekar P, Francis B. Statespace solutions to standard $H_{2}$ and $H_{\infty}$ control problems. IEEE Transactions on Automatic Control 1989;AC-34(8):831-47.

[4] Doyle, J. C., Francis, B. A. and Tannenbaum, A. R. (1992). Feedback Control Theory. Macmillan Publishing Company.

[5] Packard A, Doyle J. The complex structured singular value. Automatica 1993;29(1):71-109. 